\title{
Marie-José BÉGUELIN, Gilles CORMINBOEUF et Florence LEFEUVRE (dir.), Types d'unités et procédures de segmentation
}

\section{Christophe Benzitoun}

\section{OpenEdition}

\section{Journals}

Édition électronique

URL : https://journals.openedition.org/scolia/1514

DOI : $10.4000 /$ scolia.1514

ISSN : 2677-4224

\section{Éditeur}

Presses universitaires de Strasbourg

\section{Édition imprimée}

Date de publication : 9 juillet 2021

Pagination : 169-172

ISBN : 979-10-344-0091-1

ISSN : $1253-9708$

\section{Référence électronique}

Christophe Benzitoun, « Marie-José Béguelin, Gilles Corminboeuf et Florence Lefeuvre (dir.), Types d'unités et procédures de segmentation », Scolia [En ligne], 35 | 2021, mis en ligne le 09 juillet 2021, consulté le 14 juillet 2021. URL : http://journals.openedition.org/scolia/1514 ; DOI : https://doi.org/ 10.4000/scolia.1514

\section{(c) (i) (2)(2)}

Les contenus de la revue Scolia sont mis à disposition selon les termes de la Licence Creative Commons Attribution - Pas d'Utilisation Commerciale - Partage dans les Mêmes Conditions 4.0 International. 
SCOLIA 35/2021, p. 169-206.

Marie-José BÉGUELIN, Gilles CORMINBOEUF et Florence LEFEUVRE (dir.), Types d'unités et procédures de segmentation, Limoges, Éditions LambertLucas, collection Études linguistiques et textuelles, 2019, 272 pages.

L'ouvrage dirigé par Marie-José Béguelin, Gilles Corminboeuf et Florence Lefeuvre aborde l'épineuse question des unités linguistiques et de leur définition. Il compte 11 chapitres regroupés en trois parties:

1. les enjeux épistémologiques,

2. les modèles d'analyse et les choix de segmentation,

3. les analyses sur corpus et/ou la segmentation outillée.

Pas moins de 19 chercheurs et chercheuses y ont contribué. La langue sur laquelle portent les études est le français avec une exception pour un chapitre ayant pour objet l'espagnol oral. Les travaux oscillent entre des analyses sur corpus et des réflexions théoriques sur la pertinence et les critères définitoires des unités en vigueur, que celles-ci soient issues de la grammaire traditionnelle (mot, phrase, proposition) ou bien émanent d'approches plus récentes. Sont ainsi discutées la notion de construction mais aussi les unités alternatives proposées dans divers cadres, dont ceux de Claire Blanche-Benveniste ou du Groupe de Fribourg. La macrosyntaxe et la microsyntaxe sont donc au centre de plusieurs chapitres - et à travers elles la définition des relations de dépendance (dont la rection) et de la constituance.

C'est le développement de la linguistique de corpus qui a permis un renouvellement de ces questions et plus particulièrement les recherches portant sur des corpus considérés comme atypiques. En effet, pendant de nombreuses années, la linguistique historique de tradition française, bien que postulant la primauté de l'oral, s'est tournée vers l'écrit et la littérature. Les exemples oraux étaient souvent inventés et par voie de conséquence ne comportaient pas de contexte (voir Combettes, 2017). Avec l'arrivée des corpus informatisés, les sources de données se sont diversifiées et avec elles les problèmes de délimitation des unités. Comment procéder lorsqu'il n'y a pas de ponctuation, comme en français parlé, ou bien une ponctuation embryonnaire comme dans les copies d'élèves? C'est justement à cette question que s'attaque ce livre. 
Dans le chapitre inaugural, Pierre Le Goffic pose le problème de la tripartition entre langue, discours et texte et postule l'existence d'unités possiblement différentes dans ces trois dimensions du langage. On peut résumer le point de vue de Pierre Le Goffic de la manière suivante:

Au total, si le statut de la langue et celui du discours nont pas évolué depuis les débuts du langage, le texte apparaît dans ses différentes formes comme un produit historiquement variable, dont le statut est loin dêtre évident, peut être sujet à variations, et n'a sans doute pas retenu toute l'attention qu'il mérite. (p. 17)

Cela le pousse à privilégier les formes stables du langage, la langue par conséquent, en s'inscrivant ainsi dans les pas de Noam Chomsky. À travers un historique précis, Pierre Le Goffic émet l'hypothèse que l'unité phrase a connu un glissement de la langue vers le texte par l'intermédiaire de la grammaire scolaire et que les critiques ne s'adressent pas à la phrase en tant qu'unité de langue mais de texte, ce qui est effectivement le cas. Ce premier chapitre se termine sur l'impossibilité de trouver une unité de segmentation des textes.

Le second chapitre est dû à Dominique Legallois et porte sur la notion de construction. Il répond d'une certaine manière à Pierre Le Goffic concernant l'articulation entre la langue, le discours et le texte. À travers plusieurs exemples, Dominique Legallois défend de manière convaincante l'hypothèse que les constructions grammaticales devraient être considérées comme des schémas pré-discursifs. Il aborde de manière concrète le passage de l'usage à la compétence à travers ce que l'on appelle, dans d'autres approches, les séquences préconstruites, les routines ou encore la grammaticalisation.

Caroline Masseron, quant à elle, aborde la question des unités à travers l'analyse des écrits d'élèves. Après avoir dénoncé le caractère nocif de la notion de phrase en contexte d'enseignement, elle propose une segmentation de 4 copies d'élèves en clauses et périodes selon les principes édictés par le Groupe de Fribourg (2012). Elle défend l'idée que la segmentation est une procédure permettant de repérer les mécanismes d'encodage textuel et de mieux comprendre les erreurs des élèves. Caroline Masseron cherche à doter les enseignants d'outils adaptés afin d'améliorer les pratiques pédagogiques.

Le quatrième chapitre a été rédigé par Annie Kuyumcuyan. Cette dernière s'intéresse aux unités de discours en prenant comme référence le cadre de l'école de Genève (Roulet et alii, 2001). Elle se focalise 
plus précisément sur l'articulation entre syntaxe et discours dans les dialogues fictionnels littéraires au niveau de la co-construction des énoncés. Alain Berrendonner, de son côté, s'intéresse aux clauses nominales et propose une hypothèse disruptive. Plutôt que de tenter à tout prix de ramener les clauses nominales à des clauses verbales, comme le font de nombreux chercheurs, il pense que «ce sont les clauses nominales qui donnent la clef du sémantisme des clauses verbales» (p. 98). Ainsi Alain Berrendonner considère que les clauses nominales sont des constructions centrales en français.

Oscar Garcia Marchena aborde un sujet proche de celui d'Alain Berrendonner, à savoir les phrases averbales et les fragments. À partir du Corpus oral de référence de la langue espagnole contemporaine, il distingue les phrases averbales, qui sont des unités non elliptiques, et les fragments qui sont généralement liés au dialogue et s'appuient sur le contexte antérieur pour pouvoir être interprétés.

Le sixième chapitre est l'œuvre de Marie-José Béguelin et de Gilles Corminboeuf. Il porte sur les segments flottants et les greffes. Les auteurs recourent à la notion de métanalyse pour rendre compte des cas où l'on hésite entre une analyse sous forme d'intégration microsyntaxique ou de routine pragma-syntaxique. Ils préfèrent affronter l'indétermination de l'analyse plutôt que décider arbitrairement de classer les exemples dans l'une ou l'autre catégorie de manière arbitraire. Ils plaident pour des multi-segmentations à l'image des multi-transcriptions.

Florence Lefeuvre étudie les configurations discursives du type c'est vrai, je t'ai un peu oublié principalement dans des textes contemporains tirés de Frantext. Elle élabore une analyse sur trois plans distincts (syntaxe, sémantique et discours) afin de cerner le statut et la portée de c'est vrai ainsi que les différentes combinaisons dans lesquelles ce tour s'insère. Elle postule l'existence de ce qu'elle appelle une période discursive dans laquelle s'insère le tour c'est vrai accompagné de l'unité prédicative qui le précède ou qui le suit.

Georgeta Cislaru et Thierry Olive s'intéressent aux processus d'élaboration des textes saisis au clavier en adoptant une approche psycholinguistique. Grâce à des logiciels d'enregistrement, ils observent les unités de performance que sont les jets textuels, à savoir des portions de textes délimités par des pauses. Ils mettent au jour des fonctionnements proches de ceux que l'on retrouve en français parlé. 
Mathilde Carnol et Anne Catherine Simon se sont penchées sur les différentes réalisations des constructions verbales dans un corpus de français parlé comportant plusieurs genres de parole. Elles se sont principalement attachées à l'étude des dispositifs syntaxiques au sens de Blanche-Benveniste et alii (1990) et elles montrent que le dispositif direct est de loin le plus fréquent. Dans leur corpus de 40.000 tokens, les différents arrangements sont variés mais peu fréquents.

Le chapitre qui clôt l'ouvrage porte sur l'annotation microsyntaxique et macrosyntaxique de corpus oraux francophones et germanophones. Nathalie Rossi-Gensane et ses collègues y détaillent principalement les choix faits dans les projets Rhapsodie et Orféo afin de situer les options qu'ils ont prises pour effectuer leur annotation.

En résumé, ce livre permet de faire le point sur les recherches récentes portant sur les unités, principalement à partir de corpus. Une attention particulière est portée sur le statut des données, oscillant entre le spontané et le planifié, l'oral et l'écrit. En effet, les rendements peuvent être fort différents en fonction de la nature des données, ce qui pose le problème des unités les plus adaptées pour aborder les phénomènes liés à la variation et à l'élaboration des textes.

Christophe BENZITOUN

Université de Lorraine, ATILF UMR7118 Christophe.Benzitoun@univ-lorraine.fr

\section{Références}

BLANCHE-BENVENISTE C., BILGER M., ROUGET C. \& VAN DEN EYNDE K. (1990), Le français parlé: études grammaticales, Paris, Éditions du CNRS.

COMBETTES B. (2017), L’opposition langue parlée / langue écrite dans la linguistique historique de tradition française (1860-1930), Langages 208, 69-82.

GROUPE DE FRIBOURG (2012), Grammaire de la période, Berne, Peter Lang.

ROULET E., FILLIETTAZ L. \& GROBET A. (2001), Un modèle et un instrument d'analyse du discours, Berne, Peter Lang. 